\title{
Research on Induction Motor Vector Control System Based on Internal Model Decoupling
}

\author{
HE Hu-cheng, WANG Wen-ting ${ }^{\mathrm{a}}$, ZHU Qun and Shi Lei \\ School of Electrical and Control Engineering, Xi' an University of Science and Technology, Xi'an, China
}

\begin{abstract}
As a high-performance variable frequency control technology, vector control has been widely used in the field of AC speed regulation. However, the cross-coupling potential of the induction motor after the vector transformation still affects the system performance. Therefore, the method is studied in which stator current is decoupled to excitation component and torque component using internal model control, and the internal model decoupling stator current controller is designed based on rotor field orientation. The simulation model of induction motor vector control system based on internal model decoupling is constructed with Matlab/Simulink. The simulation result shows that the internal model controller is superior to the traditional PI controller in disturbance-rejection performance and robustness.
\end{abstract}

\section{Introduction}

Induction motor is a high-order, multi-variable, and strongly-coupled nonlinear object. In order to obtain ideal speed control performance, it is necessary to decouple the torque and flux component [1]. However, in the field of $\mathrm{AC}$ speed regulation, researchers often use the adjustment method of traditional PI. This method often has a contradiction between the rapidity and overshoot of the system when the dynamic performance requirements are high. A lot of research work has been carried out recently in the field of the decoupling method. However, feedforward decoupling method can only be fully decoupled while the reference value is exactly same as the actual stator current [2]. Sliding mode decoupling method has a "flicker" phenomenon, forcing the system to perform small-amplitude, high-frequency up and down movements along a specified state trajectory under a certain range of characteristics [3-5]. The inverse system decoupling method is proposed based on an exact mathematical model. When the controlled object parameters are changing, the control performance is less robust [6-7]. And the intelligent decoupling method is difficult to implement in engineering, which limits the wide application of this method [8].

The decoupling control of the internal model is essentially a zero-pole cancellation control method. This control method separates the uncertainties of the internal model of the controlled object to improving the robustness of the system [9]. The internal model control has high accuracy which does not require the mathematical model of the controlled object, and it can dynamically follow the given signal and eliminate the influence of unmeasured interference on the system. Meanwhile, this control strategy has a simple structure, easy in implementation and easy in setting of parameters [10].

Therefore, this paper takes the induction motor as the control object, introducing the internal model decoupling control into the mathematical model of the induction motor in the rotating coordinate system, and the internal model decoupled stator current controller of the induction motor vector control system is designed. On the basis, the vector control system based on the internal model decoupling controller and the vector control system based on the PI controller were compared and studied with Matlab/Simulink. The simulation results show that the disturbance-rejection and Robustness of internal model controller is better than PI controller.

\section{Induction motor dynamic mathematic- al model}

The voltage equation of the induction motor in a twophase rotating coordinate system based on rotor field orientation is:

$$
\left[\begin{array}{l}
u_{s d} \\
u_{s q} \\
u_{r d} \\
u_{r q}
\end{array}\right]=\left[\begin{array}{cccc}
R_{s}+p L_{s} & -\omega_{s} L_{s} & p L_{m} & -\omega_{s} L_{m} \\
\omega_{s} L_{s} & R_{s}+p L_{s} & \omega_{e} L_{m} & p L_{m} \\
p L_{m} & -\Delta \omega_{r} L_{m} & R_{r}+p L_{r} & -\Delta \omega_{r} L_{r} \\
\Delta \omega_{r} L_{m} & p L_{m} & \Delta \omega_{r} L_{r} & R_{r}+L_{r} p
\end{array}\right]\left[\begin{array}{c}
i_{s d} \\
i_{s q} \\
i_{r d} \\
i_{r q}
\end{array}\right]
$$

Where, $i_{s d}, i_{s q}$ are $d, q$ phase stator currents in a twophase rotating coordinate system. $u_{s d}, u_{s q}$ are voltages. $L_{s}$, $L_{r}, L_{m}$ are stator rotor inductance and Inter-rotor mutual inductance; $R_{s}, R_{r}$ are stator rotor resistance. $\omega_{s}$ is stator rotational magnetic field synchronous angular velocity,

$\overline{{ }^{a} \text { Wang wen ting:547531828@qq.com }}$ 
$\Delta \omega_{r}$ is rotor slip angular velocity and $p$ is differential operator.

For the squirrel-cage induction motors, the rotor is short-circuited, so $u_{r d}=u_{r q}=0$, and the voltage equation in the $d q$ coordinate system can be rewritten as:

$$
\left\{\begin{array}{l}
u_{s d}=\left(R_{\mathrm{s}}+\sigma L_{s} p\right) i_{s d}-\omega_{s} \sigma L_{s} i_{s q}+p \frac{L_{m}}{L_{r}} \psi_{r d} \\
u_{s q}=\left(R_{\mathrm{s}}+\sigma L_{s} p\right) i_{s q}+\omega_{s} \sigma L_{s} i_{s d}+\frac{\omega_{s} L_{m}}{L_{r}} \psi_{r d}
\end{array}\right.
$$

Where, $\sigma=1-L^{2}{ }_{m} / L_{s} L_{r} . \omega_{s} \sigma L_{s} i_{s d}, \omega_{s} \sigma L_{s} i_{s q}$ is coupled potential terms.

From the above equation, it can be seen that there are still cross-coupling potential terms $\omega_{s} \sigma L_{s} i_{s d}$ and $\omega_{s} \sigma L_{s} i_{s q}$ after vector transformation, and the motor parameters will directly affect the coupling term.

\section{Internal model decoupling control str- ategy}

\subsection{Internal model control basic structure}

The basic idea of the internal model control is to design a controlled object model in parallel with the controlled object. And to use the error between the two outputs feedback to the input of the controller, the error, which will form a feedback to suppress the change of parameters, model mismatch and external interference signals. Figure. 1 shows the internal model control structure.

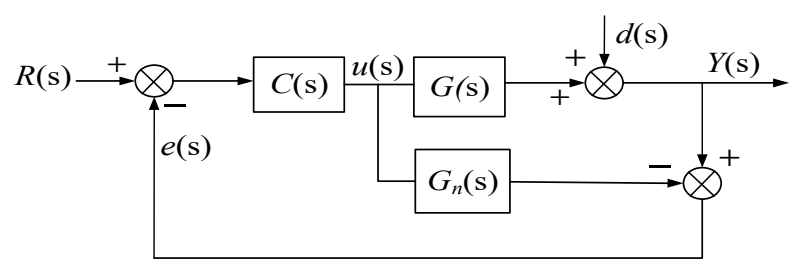

Figure 1. Internal model control structure

From Figure.1, $C(s)$ is the internal model controller. $G(s)$ is the controlled object. $G_{n}(s)$ is the estimated model of the controlled object. $e(s)$ is the feedback error signal. $R(s)$ is the input, $Y(s)$ is the output. $d(s)$ is the disturbance input. The system error and output are given by(3) and (4).

$$
\begin{aligned}
& e(s)=\frac{C(s)\left[G(s)-G_{n}(s)\right] R(s)+d(s)}{1+C(s)\left[G(s)-G_{n}(s)\right]} \\
& Y(s)=C(s) G_{n}(s) R(s)+\left[1-C(s) G_{n}(s)\right] e(s)
\end{aligned}
$$

From the above equation, when the estimation model exactly matches the controlled object, that is $G_{n}(s)=G(s)$, the output of the model is exactly same as the output of the controlled object process, which is equivalent to an open-loop system. Therefore, it is shown that the purpose of feedback is to improve the robustness of the system for a open-loop stabilization process. The feedback signal reflects the uncertainty of the controlled object and the influence of the disturbance, so the system has the nature of a closed-loop system.

\subsection{Internal model control properties}

Internal model control has three properties. Firstly, when the model is perfectly matched, the closed-loop stability of the internal model control system is only determined by the open-loop stability of each link in the forward channel. Secondly, in the ideal case, the internal model control enables unbiased tracking of the reference input. And last when certain conditions are met, internal model control has no steady-state deviation for step input and constant value interference.

\subsection{Internal mode decoupled stator current controller design}

From Figure.1, we can see that when the estimated model of the controlled object has no error with the controlled object, let $C(s)=G(s)^{-1}$, and the system can achieve full tracking input $Y(s)=R(s)$. Combined with the decoupling control principle of the internal model, the speed loop, the current loop, and the flux loop is determined, which is shown in Figure. 2.

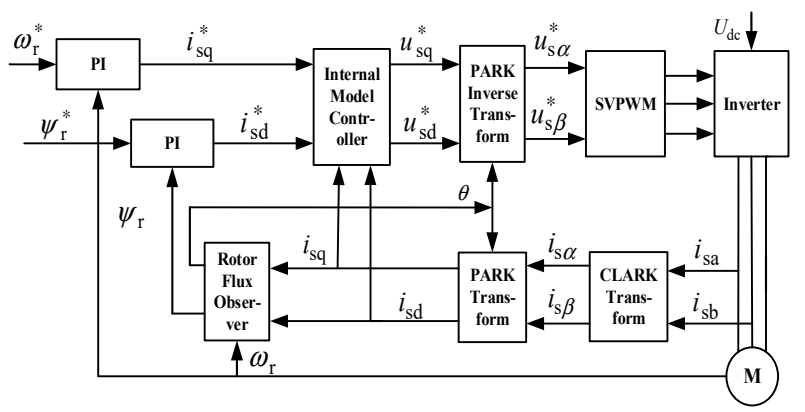

Figure. 2 Block diagram of the vector control system based on internal model decoupling

From Fig.2, there are respectively a speed control loop, a flux loop, and an internal mode current decoupling inner loop of the motor speed control system. The output of the speed loop and the flux loop are used as the internal mode decoupling $d, q$ axis current loop given, and the stator current of the motor is transformed by CLARK and PARK to obtain the current $i_{s d}$ and $i_{s q}$ in the rotating coordinate system, and $i_{s d}$ and $i_{s q}$ are used as the internal model decoupling current feedback input. The given and feedback of inner current loop by internal model decoupling controller to get $u^{*}{ }_{s d}$ and $u^{*}{ }_{s q}$. These two quantities are converted to $u_{s \alpha}^{*}$ and $u_{s \beta}^{*}$ by inverse PARK, and then sent to the SVPWM generator to generate pulses signal.

Assuming the rotor flux is constant in the process of speed regulation motor, the equation (2) can be expressed as:

$$
\left[\begin{array}{l}
u_{s d} \\
u_{s q}
\end{array}\right]=\left[\begin{array}{ccc}
R_{s}+\sigma L_{s} p & -\omega_{s} \sigma L_{s} & 0 \\
\omega_{s} \sigma L_{s} & R_{s}+\sigma_{s} p & \left(L_{m} / L_{r}\right) \omega_{s}
\end{array}\right]\left[\begin{array}{c}
i_{s d} \\
i_{s q} \\
\psi_{r d}
\end{array}\right]
$$

Substituted $u_{s q}^{\prime}=u_{s q}-\omega_{s}\left(L_{m} / L_{r}\right) \psi_{r d}$ into (5) and make the Laplace transform. Then, the motor model equation is formed as follow: 


$$
\left[\begin{array}{c}
u_{s d}(s) \\
u_{s q}^{\prime}(s)
\end{array}\right]=\left[\begin{array}{cc}
\sigma s L_{s}+R_{s} & -\omega_{s} \sigma L_{s} \\
\omega_{s} \sigma L_{s} & \sigma s L_{s}+R_{s}
\end{array}\right]\left[\begin{array}{c}
i_{s d}(s) \\
i_{s q}(s)
\end{array}\right]
$$

Equation (6) can be simplified as:

$$
Y(s)=G(s) U(s)
$$

Where:

$$
\begin{aligned}
& U(s)=\left[U_{s d} U_{s q}^{\prime}(s)\right]^{T} \\
& Y(s)=\left[i_{s d}(s) i_{s q}(s)\right]^{T} \\
& G(s)=\left[\begin{array}{cc}
\sigma s L_{s}+R_{s} & -\omega_{s} \sigma L_{s} \\
\omega_{s} \sigma L_{s} & \sigma s L_{s}+R_{s}
\end{array}\right]^{-1}
\end{aligned}
$$

In order to enhance the system robust performance, the controller is augmented by adding a filter. According to the transfer function of the induction motor, a firstorder system is usually selected. The desired filter is described as:

$$
L(s)=\frac{\lambda}{s+\lambda} I
$$

Where, $\lambda$ is the current loop bandwidth, the system closed-loop bandwidth depends only by $\lambda$. $I$ is a unit matrix. Then

$$
C(s)=\frac{L(s)}{G_{n}(s)}=\left[\begin{array}{cc}
\hat{R}_{s}+\hat{\sigma} \hat{L}_{S} s & -\omega_{S} \hat{\sigma} \hat{L}_{S} \\
\omega_{S} \hat{\sigma} L_{S} & \hat{R}_{s}+\hat{\sigma} L_{S} s
\end{array}\right] L(s)
$$

The equivalent controller expression can be described by:

$$
F(s)=\lambda\left[\begin{array}{cc}
\hat{L}_{S}\left(1+\frac{\hat{R}_{S}}{\hat{\sigma} \hat{L}_{S} S}\right) & -\omega_{S} \frac{\hat{\sigma} \hat{L}_{S}}{S} \\
\omega_{S} \frac{\hat{\sigma} \hat{L}_{S}}{S} & \hat{\sigma} \hat{L}_{S}\left(1+\frac{\hat{R}_{S}}{\hat{\sigma} \hat{L}_{S} S}\right)
\end{array}\right]
$$

In summary, the decoupling structure diagram of the internal model can be obtained as shown in Figure. 3.

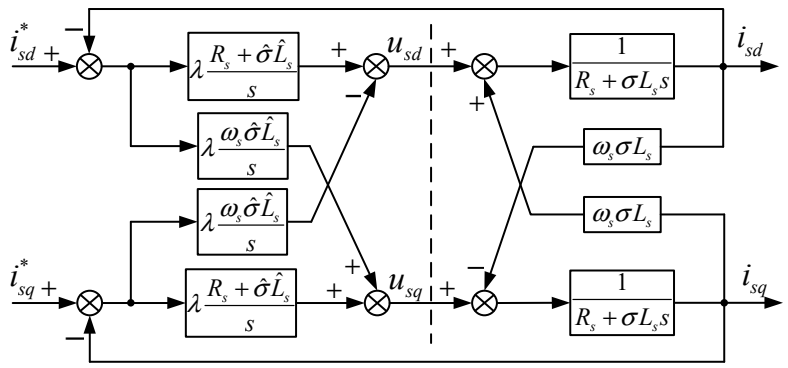

Figure. 3 Decoupling structure diagram of induction motor internal model

\section{Simulation research}

In order to verify the performance of induction motor

\begin{tabular}{|c|c|c|c|}
\hline Parameter & Value & Parameter & Value \\
\hline Rated power $P_{\mathrm{N}}$ & $3 \mathrm{~kW}$ & pole pairs $p$ & 2 \\
\hline $\begin{array}{c}\text { Rated voltage } \\
U_{\mathrm{N}} \\
\end{array}$ & $380 \mathrm{~V}$ & $\begin{array}{c}\text { stator resistance } \\
R_{\mathrm{S}} \\
\end{array}$ & $1.798 \Omega$ \\
\hline $\begin{array}{c}\text { DC bus voltage } \\
U_{\mathrm{dc}} \\
\end{array}$ & $540 \mathrm{~V}$ & rotor resistance $R_{\mathrm{r}}$ & $1.588 \Omega$ \\
\hline Rated current $I_{\mathrm{N}}$ & $6.5 \mathrm{~A}$ & $\begin{array}{c}\text { mutual } \\
\text { inductance } L_{\mathrm{m}}\end{array}$ & $0.387 \mathrm{H}$ \\
\hline $\begin{array}{c}\text { Rated frequency } \\
f_{\mathrm{N}} \\
\end{array}$ & $50 \mathrm{~Hz}$ & $\begin{array}{l}\text { stator leakage } \\
\text { inductance } L \text { s }\end{array}$ & $0.0073 \mathrm{H}$ \\
\hline Rated speed $n$ & $\begin{array}{l}1200 \mathrm{r} / \\
\mathrm{min}\end{array}$ & $\begin{array}{l}\text { rotor leakage } \\
\text { inductance Lr }\end{array}$ & $0.0077 \mathrm{H}$ \\
\hline
\end{tabular}
vector control system using the internal model decoupling controller, the system simulation model is performed. And a comparison is made between the traditional PI control system and the internal model decoupling control system. The rated parameters of the motor in simulation are shown in Table 1 .
Table 1. Simulation induction motor related parameters

The simulation is compared from the performance of the anti-load disturbance and the change performance of the anti-motor parameters. Figure. 4 shows the performance comparison between the internal model and the traditional PI system when the disturbance occurs during sudden increase or decrease. In the simulation, the operating conditions of the two systems are the same, and the rotation speed is $1200 \mathrm{r} / \mathrm{min}$. At $\mathrm{t}=0.5 \mathrm{~s}$ when sudden $20 \mathrm{~N} \cdot \mathrm{m}$ load is added. At $\mathrm{t}=1.5 \mathrm{~s}$, the load suddenly drops to no load.

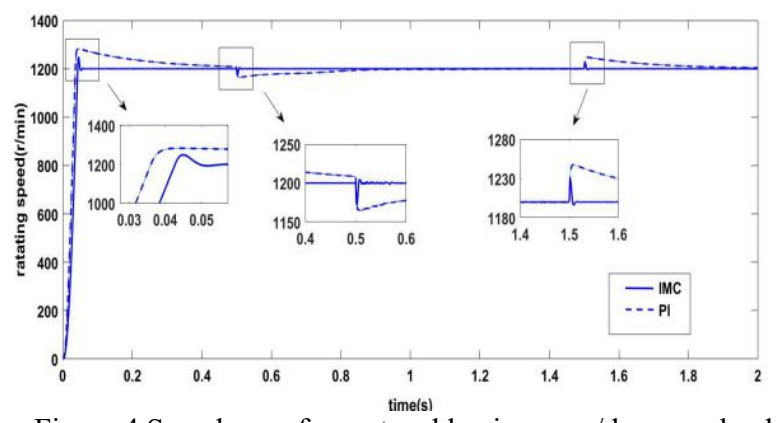

Figure.4 Speed waveform at sudden increase/decrease load

As can be seen from the figure, the overshoot of the traditional PI controller is $6.9 \%$, the internal controller is $4.1 \%$. Under load conditions, the recovery time of the traditional PI is $0.4 \mathrm{~s}$ and the the internal model controller is $0.11 \mathrm{~s}$. In the uninstall case, the recovery time of the traditional PI is $0.6 \mathrm{~s}$, and the recovery time of the internal model controller is $0.14 \mathrm{~s}$. Therefore, the internal model control system can achieve fast low overshoot and anti-load disturbance, and the effect is obviously better than the traditional PI control system.

In order to verify the robustness of the two methods, the rotor resistance $\mathrm{Rr}$ of the motor is unchanged and the rotor resistance is $1.4 \mathrm{Rr}$. Both systems operate in the same condition and start at no load under rated speed. Figure. 5 shows the change of the speed waveform after the motor rotor resistance changes. 


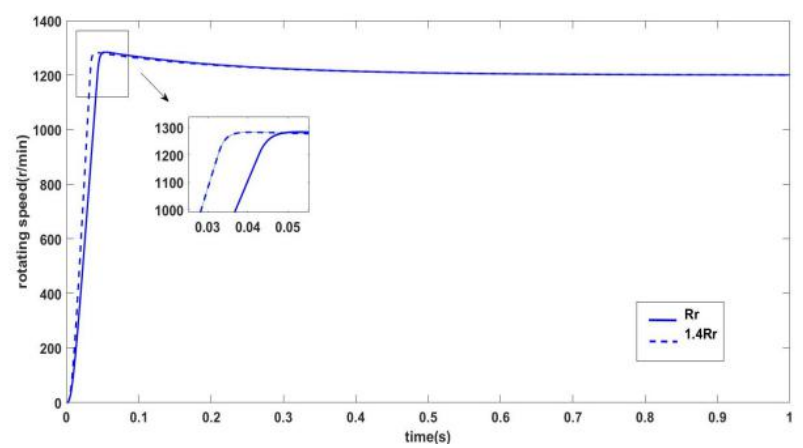

(a)PI control system speed waveform

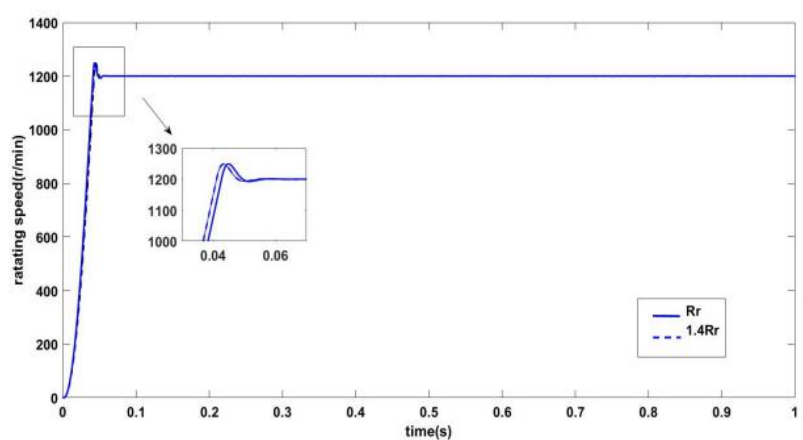

(b)Internal model control system speed waveform

Figure. 5 Motor response when rotor resistance changes

According to Figure.5, when the motor parameters have changed the dynamic performance of the PI control system changes significantly, and the internal model control system has small waveform fluctuations when the parameters change. So the internal model control system is better than the traditional PI control system.

\section{Conclusion}

The internal model controller has the advantages of small overshoot, good at disturbance rejection and robustness. The stator current decoupling control using internal model has been studied in this paper. Simulation results prove the feasibility.

\section{Acknowledgment}

The Project Supported by Natural Science Basic Research Plan in Shaanxi Province of China ( Program No. 2018JZ5014 ).

\section{References}

1. Fan Juanjuan, Luo Guangzhao,Yan Yaogang,etc. Research on Simulation of IPMSM Vector Control Based on Internal Model Decoupling. Computer Simulation,2010,27(10):347-351.

2. Zhou Zhigang. Research on decoupling control system for rotor field orientation deviation of asynchronous motor.Transactions of China Electrotechnical Society,2003(03):19-23.
3. Khoo Suiyang. Sliding Mode Control for T-S Fuzzy Systems.International Journal of Systems and Control,2007(01):21-29.

4. Xu Xuyao, Lin Hui. Position and Speed Integrated Design of Permanent Magnet Synchronous Motor Based on Dynamic Sliding Mode Control. Transactions of China Electrotechnical Society,2014,29(05):77-83.

5. ZHANG Xiaoguang, SUN Lizhi, ZHAO Ke. Nonlinear speed control for PMSM system using sliding-model control and disturbance compensation techniques. IEEE Transactions on Power Electronics,2013,28(3):1358-1365.

6. Cao Jianrong, Yu Lie, Wei Zeguo, Wang Yuhua. Research on Decoupling Control of Induction Motor Based on Inverse System Theory.Transactions of China Electrotechnical Society,1999(01):7-11.

7. Dai Xianzhong, Zhang Xinghua, Liu Guohai, Zhang Lei. Neural network inverse system linearization decoupling control of induction motor. Proceedings of the CSEE, 2004(01):112-117.

8. Harnefers L. Model Based Current Control of AC Machines Using the Internal Model Control Method.IEEE Transactions on Industry Applications, 1998,34(1):131-141.

9. ZHOU Yuanshen. Internal Model Decoupling of Induction Motor Cross-Coupling Voltage. Machine Tools \& Hydraulics, 2008(07):357-360.

10. CHEN Juan, DONG Cuiying. Composite internal model control of nonlinear systems based on neural network. Journal of Harbin University of Science and Technology, 2004(01): 17-20. 